\title{
A atuação do enfermeiro e da equipe multidisciplinar no controle da hipertensão arterial sistêmica através da educação em saúde: uma revisão integrativa
}

The nursing and multidisciplinary team in the control of systemic arterial hypertension through health education: an integrative review

El desempeño de las enfermeras y el equipo multidisciplinar en el control de la hipertensión arterial sistémica mediante la educación en salud: una revisión integrativa

Micheli Rodrigues de Oliveira ${ }^{1 *}$, Vivian Miranda Lago

\section{RESUMO}

Objetivo: Avaliar as evidências disponíveis na literatura sobre a atuação do Enfermeiro e da equipe multidisciplinar no controle da hipertensão através da educação em saúde. Métodos: Este estudo adotou a revisão integrativa da literatura como forma de pesquisa utilizando as bases de dados da Biblioteca Virtual de Saúde (BVS) e Google Acadêmico com a combinação dos descritores HAS, Enfermeiro, equipe multidisciplinar, educação em saúde. Resultados: Foram revisados sete estudos que abordavam o tema e a análise destes apontaram a importância da educação em saúde no controle da hipertensão arterial por meio do profissional Enfermeiro, assim como a abordagem da equipe multiprofissional e o apoio da família na adesão ao tratamento. Considerações finais: Infere-se que o profissional Enfermeiro e a equipe multidisciplinar, somado ao comprometimento do usuário, são os principais responsáveis pelo tratamento do paciente portador de HAS, através da educação em saúde, estimulando a prática do autocuidado dando adesão ao tratamento, e aproximando os portadores de HAS aos serviços de saúde. A continuidade dos estudos neste tema é necessária para o desenvolvimento de planejamentos estratégicos de ações básicas de saúde aos portadores de HAS.

Palavras-chave: HAS, Enfermeiro, Equipe multidisciplinar, Educação em saúde.

\section{ABSTRACT}

Objective: To evaluate the available evidence in the literature on the role of the nurse and the multidisciplinary team in the control of hypertension through health education. Methods: This study adopted an integrative review of the literature as a form of research using the databases of the Virtual Health Library (VHL) and Google Scholar with the combination of the descriptors SAH, Nursing, multidisciplinary team, health education. Results: We reviewed seven studies that addressed the topic and analyzed the importance of health education in the control of arterial hypertension through the professional nurse, as well as the approach of the multiprofessional team and the support of the family in adherence to treatment. Final considerations: It was inferred that the nurse practitioner and the multidisciplinary team, in addition to the commitment of the user, are the main responsible for the treatment of the patient with hypertension, through health education, stimulating the practice of self-care giving adherence to treatment, and approaching The holders of HBP to health services. The continuity of the studies in this subject is necessary for the development of strategic plans of basic health actions to those with SAH.

Key words: SAH, Nurse, Multidisciplinary team, Health education.

\footnotetext{
${ }^{1}$ Faculdade Vale do Cricaré (FVC), São Mateus - ES. *E-mail: michelirodrigues.be@hotmail.com
} 


\section{RESUMEN}

Objetivo: Evaluar la evidencia disponible en la literatura sobre el papel de la Enfermera y el equipo multidisciplinario en el control de la hipertensión a través de la educación en salud. Métodos: Este estudio adoptó la revisión integradora de la literatura como forma de investigación utilizando las bases de datos de la Biblioteca Virtual en Salud (BVS) y Google Scholar con la combinación de los descriptores HAS, Enfermera, equipo multidisciplinario, educación en salud. Resultados: Se revisaron siete estudios que abordaron el tema y en su análisis se señaló la importancia de la educación en salud en el control de la hipertensión arterial a través de la enfermera profesional, así como el abordaje del equipo multidisciplinario y el apoyo de la familia en la adherencia al tratamiento. Consideraciones finales: Se infiere que la Enfermera profesional y el equipo multidisciplinario, sumado al compromiso del usuario, son los principales responsables del tratamiento del paciente, a través de la educación en salud, estimulando la práctica del autocuidado dando adherencia al tratamiento y acercando a los pacientes a los servicios de salud. La continuidad de los estudios sobre este tema es necesaria para el desarrollo de planes de acciones básicas de salud para pacientes.

Palabras clave: HAS, Enfermera, Equipo multidisciplinario, Educación en salud.

\section{INTRODUÇÃO}

A Hipertensão Arterial Sistêmica (HAS) é um problema grave de saúde pública não só no Brasil, mas em todo o mundo. Atualmente, este mal tem sido um dos mais importantes fatores de risco para o desenvolvimento de doenças graves como as cardiovasculares, cerebrovasculares e renais, sendo responsável por pelo menos $40 \%$ das mortes por Acidente Vascular Cerebral (AVC), por $25 \%$ das mortes por doença arterial coronariana, e em combinação com o diabetes, $50 \%$ dos casos de insuficiência renal terminal (LOPES MCL e MARCON SS, 2009).

Dados de origem epidemiológica que foram obtidos em estudo puderam demonstrar que em todo o país as doenças de cunho cardiovascular são as principais causas de óbito na população em geral. E no Brasil, cerca de 60 a $80 \%$ dos casos de hipertensão podem ser tratados na rede primária de saúde, necessitando apenas de medidas de caráter preventivo e de promoção de saúde (BRASIL MS, 2012).

Devido às suas diversas origens, a hipertensão arterial sistêmica demonstra índices elevados e preocupantes de prevalência e os pacientes acometidos apresentam grande dificuldade de controlar essa comorbidade. Estima-se que apenas um terço das pessoas, regularmente acompanhadas em serviços de saúde tem a Pressão Arterial (PA) mantida em níveis desejáveis. Estudos prévios realizados demonstram que cerca de $95 \%$ dos casos de diabetes mellitus e hipertensão são originados por fatores genéticos e associados a fatores ambientais (MARTINS MA, et al., 2015).

Um dos mais importantes determinantes desse problema é a não adesão adequada ao tratamento medicamentoso (SANTA-HELENA ET, et al., 2010). O desconhecimento sobre o que é hipertensão arterial sistêmica, seus fatores de risco, e a importância do tratamento pode resultar em consequências irreversíveis. O principal fator de relevância da identificação e controle da hipertensão arterial sistêmica reside na redução das suas complicações, tais como: doença cerebrovascular; doença arterial coronariana; insuficiência cardíaca; doença renal crônica; doença arterial periférica e muitas outras que acometem diversas pessoas ao redor do mundo (SANT'ANA DOM, 2017).

Vários são os fatores que dificultam o controle e o tratamento da hipertensão arterial sistêmica, e entre esses, pode-se destacar a não adesão ao devido tratamento. Embora não seja um problema exclusivo da hipertensão arterial sistêmica, por ser frequente em outros regimes terapêuticos de caráter prolongado, a falta de adesão ao correto tratamento anti-hipertensivo se aprofunda em complexidade (SANTOS ZMSA e LIMA HP, 2008).

Um dos fatores que dificulta o tratamento é a ausência de sintomas na hipertensão arterial sistêmica, pois apenas metade das pessoas que sofrem de pressão alta sabe que tem a doença em questão, dessa forma 
por não apresentarem sintomas, geralmente têm a impressão de gozar dos frutos de uma boa saúde. Estas pessoas podem desencadear uma alteração na Pressão Arterial (PA) e, em conjunto, hábitos e comportamentos de saúde que favoreçam a permanência da mesma elevada. A grande maioria das pessoas toma conhecimento do diagnóstico desse problema grave quando são vítimas de alguma possível complicação como infarto, aneurisma e insuficiência renal (DE SOUZA JM, 2011).

A educação voltada a área da saúde não deve ser apenas de caráter informativo, e sim levar os usuários assim como os pacientes a refletirem sobre as bases sociais de sua vida, passando a perceber a saúde não mais como uma concessão, mas como um direito social estabelecido na Constituição Federal Brasileira de 1988 (ALVES VS, 2005).

Dessa forma, a educação em saúde constitui uma importante estratégia para propiciar conhecimento e informação aos portadores de hipertensão arterial, contribuindo de forma significativa e proveitosa para a melhoria nas condições de saúde, no emprego de hábitos e estilos de uma vida mais saudável. Estudiosos acreditam que esse modelo de educação já adotado em países desenvolvidos, como no Canadá, de forma permanente, possa ser generalizado para os diversos países, assim como as medidas gerais do controle de fatores de risco. (CAMPBELL NRC, et al., 2003; MCLISTER FA, 2006; ONYSKO J, et al., 2006).

Mesmo com a melhoria impressionante dos indicadores, os pesquisadores comentam que há muito a ser feito e descoberto em relação ao controle e à promoção da saúde de pessoas com hipertensão arterial sistêmica, já que um terço desta população mantém a sua hipertensão não controlada ou ainda, não diagnosticada (CAMPBELL NRC, et al., 2003; MCLISTER FA, 2006; ONYSKO J, et al., 2006).

Estudos apontam a grande importância dos profissionais de saúde em relação à educação dos pacientes em saúde. O ideal é que os profissionais da saúde possuam qualidades de boa comunicação em primeiro contato, longitudinalidade, integralidade, coordenação de cuidados, focalização na família, orientação comunitária e competência cultural (MAIA LG, et al., 2020).

Em termos práticos, é possível observar a importância do enfermeiro como mediador, possibilitando ao paciente a verbalização de seus problemas. O enfermeiro é identificado não somente como um profissional, mas como alguém de confiança com quem se pode compartilhar os problemas e situações de natureza física, social, familiar, econômica e emocional. $\mathrm{Na}$ grande parte das vezes, os hipertensos desejam não só esclarecimentos para suas dúvidas, mas também alguém que amenize seus anseios. Diversos estudos comprovam a contribuição do enfermeiro em programas de orientação de portadores de hipertensão, aumentando o índice de adesão ao tratamento (JARDIM PCBV, 2004).

Por isso, o papel do profissional Enfermeiro é importante em sua implementação. Torna-se imprescindível que o Enfermeiro conheça atitudes, crenças, percepções, pensamentos e práticas desenvolvidas no cotidiano pelo portador de HAS para que possa incentivá-lo de forma positiva e esclarecedora a uma participação ativa em seu tratamento (SANT'ANA DOM, 2017).

O presente estudo teve como objetivo avaliar as evidências que estão disponíveis na literatura sobre a atuação do desse profissional e da equipe multidisciplinar no controle da HAS através da educação em saúde.

\section{MÉTODOS}

O presente estudo de abordagem qualitativa baseado na revisão da literatura objetivou pontuar a atuação do enfermeiro junto à equipe multidisciplinar no controle da HAS. Adotou-se a revisão integrativa da literatura, uma vez que ela contribui para o processo de sistematização e análise dos resultados, visando a compreensão de determinado tema, a partir de outros estudos independentes.

Para tanto, foram adotadas as seguintes etapas indicadas para a constituição da revisão integrativa da literatura: estabelecimento da hipótese e objetivos da revisão integrativa; estabelecimento de critérios de inclusão e exclusão de artigos (seleção da amostra); definição das informações a serem extraídas dos artigos selecionados; análise dos resultados e discussão. 
A questão norteadora desta revisão integrativa foi: Qual a importância da atuação do Enfermeiro e equipe multidisciplinar no controle da HAS através da educação em saúde?

Para a seleção dos artigos foram utilizadas a Biblioteca Virtual de Saúde (BVS), onde contém as principais bases de dados em Ciências de Saúde, como a Literatura Latino-Americana e do Caribe em Ciências da Saúde (LILACS), Scientific Electronic Library Online (SciELO) e Medical Literature analysis and Retrievel System Online (Medline), e google acadêmico, por serem bibliotecas eletrônicas de acesso livre e supõe-se que tenham boas possibilidades de impacto nos meios acadêmicos e profissionais.

Os critérios de inclusão dos artigos definidos para a presente revisão integrativa foram: todas as categorias de artigo (original, revisão de literatura, reflexão, atualização, relato de experiência etc), artigos publicados em português, com os resumos disponíveis nas bases de dados selecionadas, no período compreendido entre 2007 e 2019. Os critérios de exclusão foram: artigos não presentes nas bases de dados utilizadas, artigos em línguas estrangeiras, e artigos com data de publicação anterior à 2007, como pode ser observado abaixo (Figura 1). 
Figura 1 - Fluxograma do processo de seleção dos artigos utilizados para o trabalho de revisão.

Busca estratégica nas bases de dados

(LILACS, SciELO, Medline e Scholar)

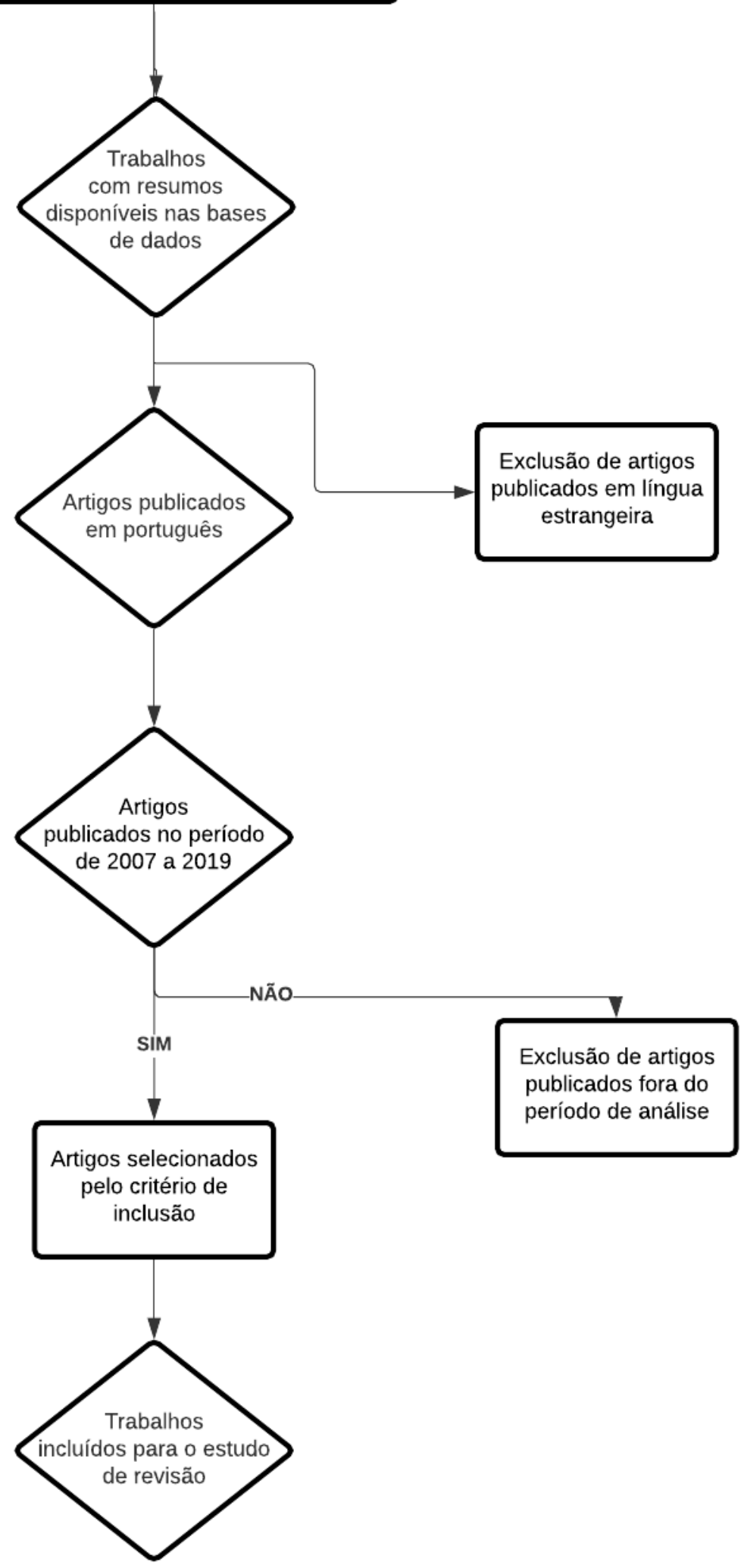

Fonte: Oliveira MR e Lago VM, 2021. 
As palavras-chave utilizadas na busca para o estudo foram: Enfermeiro, Hipertensão arterial, equipe multidisciplinar e educação em saúde. A busca foi realizada pelo acesso on-line e, chegou-se ao total de 38 artigos, que após a inserção dos critérios de inclusão foram selecionados 07 a qual responderam ao propósito do trabalho.

A busca pelos artigos foi realizada no mês de junho de 2020 e utilizou-se palavras chaves de busca, com os descritores combinados: Enfermeiro AND Hipertensão arterial, equipe multidisciplinar AND Hipertensão arterial e educação em saúde AND Hipertensão arterial.

Para a coleta de dados dos artigos que foram incluídos na revisão integrativa, foi desenvolvido um quadro sinóptico com a síntese dos artigos que atenderam aos critérios de inclusão.

\section{RESULTADOS}

Os artigos foram analisados e comparados de acordo com os objetivos e resultados de cada um dos estudos de forma a relacionar os diferentes levantamentos acerca do tema educação em saúde presentes na literatura (Quadro 1). 
Quadro 1 - Síntese dos artigos selecionados contendo objetivos, resultados e conclusões de cada um.

\begin{tabular}{|c|c|c|c|c|}
\hline Autor e Ano & Título Do Artigo & Objetivo & Resultados & Conclusões \\
\hline $\begin{array}{l}\text { TOLEDO MM, } \\
\text { et al., } 2007 .\end{array}$ & $\begin{array}{l}\text { Educação em saúde no } \\
\text { enfrentamento da } \\
\text { hipertensão arterial: } \\
\text { uma nova ótica para um } \\
\text { velho problema }\end{array}$ & $\begin{array}{l}\text { Levantar a produção científica } \\
\text { multiprofissional e da enfermagem } \\
\text { sobre a prática de educação em } \\
\text { saúde nos serviços de saúde, } \\
\text { referentes ao enfrentamento da } \\
\text { hipertensão. }\end{array}$ & $\begin{array}{l}\text { Considerando que foi realizada busca em base de } \\
\text { dados de produção específica da enfermagem, } \\
\text { observa-se que a categoria profissional de } \\
\text { enfermagem se sobressai frente ao desenvolvimento } \\
\text { dos trabalhos. Este dado pode traduzir uma } \\
\text { preocupação maior dos enfermeiros com a temática } \\
\text { em questão. Todavia, a perspectiva da promoção da } \\
\text { saúde requer multidisciplinariedade, integralidade, } \\
\text { bem como intersetor alidade. }\end{array}$ & $\begin{array}{l}\text { Conclui-se que as experiências } \\
\text { educativas com usuários portadores de } \\
\text { hipertensão são incipientes e poucos } \\
\text { artigos se reportam à perspectiva de } \\
\text { formação da "consciência crítica" sobre } \\
\text { saúde. }\end{array}$ \\
\hline $\begin{array}{l}\text { FAVA SMCL, } \\
\text { et al., } 2010 .\end{array}$ & $\begin{array}{l}\text { Diagnóstico de } \\
\text { enfermagem e proposta } \\
\text { de intervenções para } \\
\text { clientes com } \\
\text { hipertensão arterial. }\end{array}$ & $\begin{array}{l}\text { Identificar características } \\
\text { definidoras e fatores } \\
\text { relacionados para o diagnóstico de } \\
\text { enfermagem conhecimento } \\
\text { deficiente e estabelecer } \\
\text { intervenções de enfermagem. }\end{array}$ & $\begin{array}{c}\text { Ao avaliar o conhecimento dos sujeitos sobre a } \\
\text { patologia, foi observado que } 17(85 \%) \text { desconheciam o } \\
\text { significado de pressão alta, mencionando conceitos } \\
\text { vagos e errôneos. Constatou-se que, em } 17(85 \%) \\
\text { sujeitos, os fatores relacionados são atribuídos } \\
\text { principalmente à falta de interesse em aprender. Este } \\
\text { dado merece destaque, pois o conhecimento, as } \\
\text { crenças e as percepções que a maioria dos portadores } \\
\text { de hipertensão arterial têm sobre a doença fortalecem } \\
\text { a adesão } \\
\text { ao tratamento anti-hipertensivo. }\end{array}$ & $\begin{array}{c}\text { A Sistematização da Assistência de } \\
\text { Enfermagem contribui de forma } \\
\text { significativa para melhorias no processo } \\
\text { do autocuidado, ao propiciar } \\
\text { conhecimentos com vistas à } \\
\text { conscientização quanto ao seu estado } \\
\text { de saúde e à necessidade de } \\
\text { mudanças, o que facilita a adesão ao } \\
\text { tratamento. }\end{array}$ \\
\hline $\begin{array}{c}\text { MENEZES } \\
\text { AGMP e } \\
\text { GOBBI D, } \\
2010 .\end{array}$ & $\begin{array}{l}\text { Educação em saúde e } \\
\text { Programa de Saúde da } \\
\text { Família: atuação da } \\
\text { enfermagem na } \\
\text { prevenção de } \\
\text { complicações } \\
\text { em pacientes } \\
\text { hipertensos }\end{array}$ & $\begin{array}{l}\text { Ressaltar a importância da } \\
\text { implantação de métodos } \\
\text { preventivos em pacientes } \\
\text { hipertensos para evitar } \\
\text { complicações; propor intervenções } \\
\text { de enfermagem; identificar } \\
\text { ações de educação em saúde no } \\
\text { Programa de Saúde da Família } \\
\text { (PSF) para familiares e pacientes. }\end{array}$ & $\begin{array}{l}\text { O enfermeiro com sua função educadora deve integrar } \\
\text { as ações educativas tanto para o paciente como para } \\
\text { sua família, afinal uma melhora na qualidade de vida é } \\
\text { importante para toda a população e não apenas para } \\
\text { os hipertensos. }\end{array}$ & $\begin{array}{l}\text { Promover um estilo de vida saudável } \\
\text { deve ser prioridade da equipe de saúde } \\
\text { para os indivíduos hipertensos, pois } \\
\text { desta forma serão evitadas } \\
\text { complicações e futuras internações. } \\
\text { Com isso, os gastos do SUS serão } \\
\text { menores e o paciente terá uma vida } \\
\text { mais saudável, uma vez que o risco de } \\
\text { se desenvolver demais co-morbidades } \\
\text { será diminuído. }\end{array}$ \\
\hline
\end{tabular}




\begin{tabular}{|c|c|c|c|c|}
\hline Autor e Ano & Título Do Artigo & Objetivo & Resultados & Conclusões \\
\hline $\begin{array}{l}\text { GUEDES NG, } \\
\text { et al., } 2012 .\end{array}$ & $\begin{array}{l}\text { Intervenções de } \\
\text { enfermagem } \\
\text { relacionadas à } \\
\text { promoção da saúde em } \\
\text { portadores de } \\
\text { hipertensão }\end{array}$ & $\begin{array}{l}\text { Identificar as competências de } \\
\text { promoção da saúde em } \\
\text { intervenções de enfermagem em } \\
\text { portadores de hipertensão arterial }\end{array}$ & $\begin{array}{l}\text { Os domínios de competência para a prática de } \\
\text { promoção da saúde mais evidenciados foram catalisar } \\
\text { mudanças, Avaliação das necessidades e Parcerias }\end{array}$ & $\begin{array}{l}\text { Os resultados deste estudo poderão } \\
\text { subsidiar as competências dos } \\
\text { enfermeiros para a prática da promoção } \\
\text { da saúde em portadores de hipertensão } \\
\text { arterial. }\end{array}$ \\
\hline $\begin{array}{l}\text { COSTA YF, et } \\
\text { al., } 2014 .\end{array}$ & $\begin{array}{l}\text { O papel educativo do } \\
\text { enfermeiro na adesão } \\
\quad \text { ao } \\
\text { tratamento da } \\
\text { Hipertensão Arterial } \\
\quad \text { Sistêmica: } \\
\text { revisão integrativa da } \\
\quad \text { literatura }\end{array}$ & $\begin{array}{l}\text { Descrever o papel educativo do } \\
\text { enfermeiro na adesão ao } \\
\text { tratamento da Hipertensão } \\
\text { Arterial Sistêmica. }\end{array}$ & $\begin{array}{c}\text { Os resultados mostraram que a intervenção nos } \\
\text { fatores modificáveis da HAS se fundamenta na } \\
\text { mudança dos hábitos e estilos de vida que contribuem } \\
\text { para o agravo da doença. Para tanto, a educação em } \\
\text { saúde deve ser priorizada pelo enfermeiro, enfatizando } \\
\text { a importância de hábitos saudáveis. }\end{array}$ & $\begin{array}{l}\text { Concluiu-se que os enfermeiros podem } \\
\text { estimular a prática do autocuidado. As } \\
\text { práticas educativas devem atender às } \\
\text { necessidades individuais e familiares } \\
\text { para maior adesão ao tratamento não } \\
\text { medicamentoso e ao medicamentoso, } \\
\text { por parte do portador de HAS. O } \\
\text { enfermeiro pode contribuir de forma } \\
\text { significativa para a melhoria nas } \\
\text { condições de saúde e qualidade de vida } \\
\text { do portador de HAS. }\end{array}$ \\
\hline $\begin{array}{l}\text { MOURA DJM, } \\
\text { et al., } 2011 .\end{array}$ & $\begin{array}{l}\text { Cuidado de } \\
\text { enfermagem ao cliente } \\
\text { com hipertensão: uma } \\
\text { revisão bibliográfica }\end{array}$ & $\begin{array}{c}\text { Identificar as práticas de cuidado } \\
\text { de enfermagem ao hipertenso nas } \\
\text { produções científicas dos últimos } \\
\text { dez anos. }\end{array}$ & $\begin{array}{l}\text { Nos resultados mostra a predominância da consulta de } \\
\text { enfermagem como prática mais utilizada nas } \\
\text { produções, por meio da Sistematização da Assistência } \\
\text { de Enfermagem e a Educação em Saúde com } \\
\text { abordagens individuais, além de visitas domiciliárias na } \\
\text { abordagem familiar. Acredita-se que a sistematização, } \\
\text { na organização do cuidado, contribuirá sobremaneira à } \\
\text { adesão ao tratamento anti-hipertensivo. }\end{array}$ & $\begin{array}{l}\text { Conclui-se que para uma ação efetiva } \\
\text { na prevenção e no controle da } \\
\text { hipertensão, é necessário aliar } \\
\text { educação, trabalho e fator social, não } \\
\text { em âmbito individual e sim coletivo, } \\
\text { desafiando os enfermeiros assistenciais } \\
\text { a refletirem acerca das práticas de } \\
\text { cuidado de enfermagem à clientela } \\
\text { hipertensa, no sentido de contribuir para } \\
\text { o seu controle e qualidade de vida dos } \\
\text { pacientes e de seus familiares. }\end{array}$ \\
\hline $\begin{array}{l}\text { LOPES MCL, } \\
2009 .\end{array}$ & $\begin{array}{l}\text { O autocuidado em } \\
\text { indivíduos com } \\
\text { hipertensão arterial: um } \\
\text { estudo bibliográfico }\end{array}$ & $\begin{array}{l}\text { Identificar a forma como o } \\
\text { Autocuidado do hipertenso tem } \\
\text { sido abordado } \\
\text { na literatura nacional. }\end{array}$ & $\begin{array}{l}\text { Os estudos valorizaram a abordagem da equipe } \\
\text { interdisciplinar para observar e compreender a visão } \\
\text { do indivíduo sobre a hipertensão arterial e atuar no } \\
\text { suporte educacional da pessoa com hipertensão } \\
\text { arterial para a realização do autocuidado. Considera- } \\
\text { se a família um agente de suporte para o cuidado a } \\
\text { essas pessoas. }\end{array}$ & $\begin{array}{c}\text { Profissionais precisam modificar suas } \\
\text { condutas, principalmente para o } \\
\text { aconselhamento em saúde, repensando } \\
\text { sua } \\
\text { prática enquanto instrumento } \\
\text { responsável pelo controle da doença. }\end{array}$ \\
\hline
\end{tabular}

Fonte: Oliveira MR e Lago VM, 2021; dados extraídos da Biblioteca Virtual de Saúde (BVS). 


\section{DISCUSSÃO}

Os agentes profissionais de saúde são responsáveis pela comunicação direta com os pacientes e pelo devido conhecimento acerca da condição de saúde de cada um dos enfermos. Dessa forma, a abordagem multiprofissional, por meio dos diversos saberes que os profissionais envolvidos possuem em conjunto com a realização de grupos de apoio educativo têm se mostrado um instrumento de grande valor no controle da doença hipertensiva, por se tratar de uma forma de interação entre os profissionais da saúde e os usuários do sistema de saúde, fazendo com que estes possam refletir e expor a sua realidade, podendo observar os problemas mais comuns entre eles, trocar experiências e propor mudanças de hábitos e estilos de vida (TOLEDO MM, et al., 2007).

A educação em saúde não deve ser apenas de caráter informativo, e sim levar os usuários a refletirem sobre as bases sociais de sua vida, passando a perceber a saúde não mais como uma concessão, mas como um direito social estabelecido na Constituição Federal Brasileira de 1988 (FAVA SMCL, et al., 2010).

O papel do Enfermeiro é importante em sua implementação. Torna-se imprescindível que este profissional conheça atitudes, crenças, percepções, pensamentos e práticas desenvolvidas no cotidiano pelo portador de HAS para que possa incentivá-lo a uma participação ativa em seu tratamento (MENEZES AMP e GOBBI D, 2010). A educação em saúde é a melhor maneira para se mudar hábitos que possam ser nocivos à saúde, e no caso da prevenção, as atividades educativas são essenciais. A educação em saúde, então, passa a ser essencial para melhorar a qualidade da assistência de enfermagem (GUEDES NG, et al., 2012).

O enfermeiro junto à equipe multidisciplinar deve acompanhar o usuário do sistema de saúde ou realizar intervenções coletivas considerando o perfil da comunidade assistida. Para uma prevenção adequada, devese sempre enfocar a orientação sobre os riscos da doença. Nessa perspectiva, a educação em saúde é uma atividade destinada a melhorar a saúde pelo aumento do conhecimento teórico e prático das pessoas, assim como favorecer a mudança de atitudes das pessoas para comportamentos mais saudáveis e mais qualidade de vida (COSTA YF, et al., 2014).

A presença de uma equipe multidisciplinar contribui de forma eficaz na adesão ao tratamento da HAS. Dessa forma, é de extrema importância a atuação de uma equipe em busca da prevenção de complicações em pacientes hipertensos. Cabe aos profissionais estarem devidamente orientados sobre as características da doença assim como as formas de tratamento, objetivando melhor domínio sobre a doença (COSTA YF, et al., 2014; TOLEDO MM, et al., 2017).

Nesse sentindo, é de grande importância que o profissional enfermeiro possa exercer o seu papel de forma a acolher as muitas eventualidades, circunstâncias, hábitos e as diferentes necessidades que norteiam seus pacientes, como a condição de vida, as dificuldades inerentes às diversas classes sociais e até mesmo questões que vão além do âmbito hospitalar e que podem envolver muitos riscos ao paciente e comprometer a sua saúde e bem-estar plenas (PÓVOA R, 2020).

Estudos mostram, por exemplo, que a raça/etnia negra é a mais acometida pela incidência e mortalidade por doenças cerebrovasculares, como a hipertensão arterial sistêmica. Do ponto de vista biológico-histórico, estudos apontam que pessoas negras e pardas podem apresentar mais a hipertensão devido ao fenótipo expresso em relação à herança genética destes brasileiros com os negros escravizados vindos do continente africano nos navios negreiros, onde os escravos que mais sobreviviam eram os com capacidade de armazenar mais sais e água no organismo diante da privação de alimento e água. Todavia, existem lacunas nestes estudos que impossibilitam a compreensão completa destes fatores (PÓVOA R, 2020).

E ainda, o maior número de pardos e negros na amostra presente pode ter sido dado pelo fato de que de pardos e negros são os usuários mais frequentes do Sistema de Saúde Pública no Brasil pois possuem renda inferior quando comparados a brancos (PÓVOA R, 2020).

Outro fator de risco é o tabagismo que é apontado como um grande vilão para uma série de doenças, como o câncer, doenças cardiovasculares, pulmonares e também para o agravamento da condição de hipertensão (JOSÉ BPS, et al., 2017). Estudos apresentados por De Sousa MG (2015) demonstram que o tabagismo ocasiona um desequilíbrio no sistema nervoso autonômico e a exposição à fumaça do tabaco gera 
a ativação do sistema nervoso simpático, o qual é sensível a respostas à agentes químicos no metabolismo e responsável por enviar sinais ao sistema nervoso central, causando, assim, respostas inibitórias ou excitatória.

De Sousa MG (2015) expõe ainda que estímulos mecânicos resultantes de contrações musculares no sistema cardiovascular e pulmonar (como em artérias e vasos) resulta no envio de sinais ao sistema nervoso central, que responde a fim de diminuir a pressão arterial causada. Dessa forma, o preparo do profissional da saúde e seu conhecimento sobre o tema é fundamental para uma abordagem de sucesso frente ao paciente, principalmente ao implementar estratégias de efetivação de educação em saúde.

Assim como o tabaco, o álcool é considerado um agravante no que diz respeito à hipertensão arterial. No Brasil, estudos apontam que a prevalência média de risco no consumo de bebidas alcoólicas é alta em adultos acima de 18 anos (SILVA APD e LARA RT, 2019).

Estudos realizados pela Vigilância de Fatores de Risco e Proteção para Doenças Crônicas por Inquérito Telefônico, Vigitel (BRASIL, 2014), elucidam que uma porcentagem média dos brasileiros adultos consome abusivamente bebidas alcóolicas, e Brites RMR e Abreu ÂMM (2014) apresentam em seus estudos que 32,5\% dos adultos da cidade do Rio de Janeiro consomem álcool em excesso. Em contrapartida, Dias JRP et al. (2019) observa em sua pesquisa que um décimo dos pacientes idosos com hipertensão faz consumo de álcool, e apenas metade deles de fato nunca consumiram bebidas alcoólicas.

Dias JRP, et al. (2019) ainda faz análises que mostram que 37,5\% dos que consumiam/consomem álcool tiveram essa prática por um período de 40 a 50 anos, 37,5\% consumiram/consomem por 20 a 29 anos. Tais fatos podem ser explicados pois acredita-se que idosos diminuem ou cessam a ingestão de álcool na velhice pois muitos são acometidos por doenças crônicas.

Outro fator de risco agravante na vida dos pacientes é o sedentarismo. O hábito de realizar atividades físicas traz à saúde diversos benefícios e melhor qualidade de vida. No caso de pessoas com hipertensão arterial, este hábito se torna ainda mais importante, uma vez que a prática de exercícios físicos diminui consideravelmente os riscos de acidentes coronários causados pela hipertensão (NOGUEIRA IC, et al., 2012).

Além de que, estudos realizados por Merquiades JH, et al. (2009) mostram que pessoas com hábitos regulares de práticas de atividades físicas apresentam condições melhores quanto ao agravo de doenças e melhor resistência. De Andrade DV (2019) aborda em seu estudo os diferentes benefícios para a saúde dos pacientes, a depender de qual atividade se é realizada, e que em todas elas, é possível observar a normalização da pressão arterial em hipertensos.

O tratamento da hipertensão também pode ser realizado através da manipulação de fármacos produzidos e recomendados para pacientes hipertensos, todavia estes produtos medicamentosos muitas vezes causam diversos efeitos colaterais, como dor de cabeça, tontura, fadiga, e variação da frequência cardíaca, como apresentado por Malachias MVB, et al. (2003) e corroborado por Netto RORF (2017). Por isso, o uso de medicação não é, muitas vezes, o mais recomendado por especialistas para o tratamento da hipertensão devido ao fato que esta é uma condição crônica. Dessa forma, tem sido cada vez mais recomendado que haja intervenção dos profissionais de saúde nas medidas de incentivo aos pacientes com hipertensão para a prática de atividades físicas objetivando melhor qualidade de vida.

E, por conseguinte a idade dos indivíduos tem sido vista como um fator limitante à qualidade de vida, pois esse processo natural acaba por limitar e dificultar atividades que exigem mais esforço físico e mental. $O$ fato apresentado é de grande relevância, pois como visto, com o passar da idade a probabilidade de que haja o desenvolvimento de hipertensão é existente. A predominância do alto índice de negativas sobre a saúde pode estar associada aos sintomas da hipertensão e de ainda outras doenças associadas, levando em consideração também a idade avançada dos participantes. A hipertensão contribui para a ocorrência de desconfortos e prejudica a qualidade de vida, o que pode explicar o fato de que a maior parte dos entrevistados tenham classificado sua saúde em relação à idade como regular, já que possuem hipertensão (MOURA DJM, et al., 2011). 
Assim, faz-se necessário o desenvolvimento de estratégias de cuidado que contemplem os diversos elementos envolvidos no processo de adoecimento da hipertensão arterial: as expressivas transformações na vida dos indivíduos nas esferas emocional, familiar, social e econômica, considerando que a maior parte se constitui de usuários do Sistema Único de Saúde (SUS), dentre os quais estão embutidas dificuldades socioeconômicas e culturais que podem tornar-se empecilhos à adesão terapêutica adequada. Esse cuidado deve ser contextualizado às necessidades do indivíduo e permeado pela noção de autonomia, com vistas à produção de postura ativa na adesão (MOURA DJM, et al., 2011).

Acredita-se que a adesão ao tratamento está atrelada ao papel educacional do profissional junto ao cliente, no que se refere à orientação do autocuidado, e essa relação enfermeiro-paciente é fundamental para que ocorram mudanças que colaborem na manutenção ou recuperação da saúde a partir do autocuidado. Considera-se neste contexto a família como um agente de suporte para o cuidado aos pacientes hipertensos (LOPES MCL, et al., 2009).

O enfermeiro, como parte integrante da equipe de saúde, assume a corresponsabilidade das ações do cuidado para a promoção da saúde e prevenção de riscos e agravos dessa doença, como no controle e acompanhamento do portador de HAS. Por meio do conhecimento científico e de seu papel de educador, ele tem a possibilidade de instrumentalizar o portador da doença para o tratamento, melhorando sua qualidade de vida (TOLEDO MM, et al., 2007).

\section{CONSIDERAÇÕES FINAIS}

O profissional Enfermeiro e sua equipe multidisciplinar, juntamente ao comprometimento do usuário são os principais responsáveis pelo sucesso no tratamento e recuperação do paciente portador da HAS. Este dispõe de conhecimento que pode ser compartilhado com os portadores de HAS através da introdução à educação em saúde, o que estimula a prática do autocuidado efetivando a adesão ao tratamento devido e aproximando os portadores de HAS aos serviços públicos de saúde (profissional/paciente). O presente estudo, apesar da derradeira limitação de ter como critério básico os estudos na língua portuguesa e da pouca literatura encontrada que retrata o tema estudado, revelou o papel educativo do enfermeiro e as ações benéficas para a adesão ao tratamento da HAS.

\section{REFERÊNCIAS}

1. ALVES VS. Um modelo de educação em saúde para o Programa Saúde da Família: pela integralidade da atenção e reorientação do modelo assistencial. Interface-Comunicação, saúde, educação, 2005; 9(16): 39-52.

2. BRASIL, Ministério da Saúde. Saúde Brasil 2013: uma análise da situação de saúde e das doenças transmissíveis relacionadas à pobreza. 2014. Disponível em: http://svs.aids.gov.br/dantps/centrais-deconteudos/publicacoes/saude-brasil/saude-brasil-2013-uma-analise-da-situacao-de-saude-e-das-doencastransmissiveis-relacionadas-a-pobreza.pdf. Acessado em: 02 de maio de 2019.

3. BRASIL. Ministério da Saúde. Política Nacional de Atenção Básica. 2012. Disponível em: http://189.28.128.100/dab/docs/publicacoes/geral/pnab.pdf. Acessado em: 25 de junho de 2019.

4. BRASIL. Rede Intergerencial de Informações para Saúde (RIPSA). DATASUS. Prevalência de Hipertensão Arterial. Vigilância de Fatores de Risco e Proteção para Doenças Crônicas por Inquérito Telefônico (VIGITEL). 2014. Disponível em: <http://tabnet.datasus.gov.br/cgi/ dh.exe?idb2012/g02.def>. Acessado em: 26 de junho de 2019.

5. BRITES RMR, ABREU ÂMM. Padrão de consumo de bebidas alcoólicas entre os trabalhadores e perfil socioeconômico. Acta Paulista de Enfermagem, 2014; 27(2): 93-99.

6. CAMPBELL NRC, et al. Tendências temporais nas prescrições de medicamentos anti-hipertensivos no Canadá antes e depois da introdução do Programa Canadense de Educação em Hipertensão. J. Hipertensão, EUA, 2003; 21(8): $1591-1597$.

7. COSTA YF, et al. O papel educativo do enfermeiro na adesão ao tratamento da Hipertensão Arterial Sistêmica: revisão integrativa da literatura. O mundo da saúde, 2014; 38(4): 473-481.

8. DE ANDRADE DV. Hipertensão arterial sistêmica e atividade física: orientações fisioterapêuticas para exercícios físicos. Fisioterapia Brasil, 2019; 3(2): 90-99.

9. DE SOUSA MG. Tabagismo e Hipertensão arterial: como o tabaco eleva a pressão. Rev. bras. hipertens, 2015; 7883.

10. DE SOUZA JM. Perfil do paciente idoso atendido no programa hiperdia do centro de saúde dois de abril do município de Ji-Paraná/RO/Profile of elderly patient in programme hiperdia attention of health center dois de abril of the city JiParaná/RO. Revista Pesquisa \& Criação, 2011; 10(2): 189-201. 
11. DIAS JRP, et al. Análise do perfil clínico-epidemiológico dos idosos portadores de hipertensão arterial sistêmica nas microáreas 4, 6 e 7 da USF tenoné. Brazilian Journal of Health Review, 2019; 2(1): 2-41.

12. FAVA SMCL, et al. Diagnóstico de enfermagem e proposta de intervenções para clientes com hipertensão arterial. Rev. enferm. UERJ, 2010; 536-540.

13. GUEDES NG, et al. Intervenções de enfermagem relacionadas à promoção da saúde em portadores de hipertensão. Acta Paulista de Enfermagem, 2012; 25(1): 151-156.

14. JARDIM PCBV, et al. A abordagem não medicamentosa do paciente com hipertensão arterial. Pierin AMG. Hipertensão arterial: uma proposta para o cuidar. Barueri: Manole, 2004; 119-38.

15. JOSÉ BPS, et al. Mortalidade e incapacidade por doenças relacionadas à exposição ao tabaco no Brasil, 1990 a 2015. Revista Brasileira de Epidemiologia, 2017; 20: 75-89.

16. LOPES MCL, et al. O autocuidado em indivíduos com hipertensão arterial: um estudo bibliográfico. Revista Eletrônica de Enfermagem, 2009; 10(1).

17. LOPES MCL, MARCON SS. La hipertensión arterial y la familia: la necesidad del cuidado familiar. Revista da Escola de Enfermagem da USP, 2009; 43(2): 343-350.

18. MAIA LG, et al. A qualidade de serviços de atenção primária, a formação profissional e o Programa Mais Médicos em uma região de saúde do sudoeste goiano. Revista Brasileira de Epidemiologia, 2020; 23-214.

19. MALACHIAS MVB, et al. VII Diretrizes Brasileiras de Hipertensão, HIPERTENSÃO, São Paulo, 2003; 10(2): $134-139$.

20. MARTINS MA, et.al. Clínica Médica, São Paulo: Manole, 2015.

21. MCLISTER FA. Programa Educativo de Hipertensão Canadense: uma iniciativa canadense única. Canadian Journal Cardiology, 2006.

22. MENEZES AGMP, GOBBI D. Educação em saúde e Programa de Saúde da Família: atuação da enfermagem na prevenção de complicações em pacientes hipertensos. Mundo saúde, 2010; 34(1): 97-102.

23. MERQUIADES JH, et al. A importância do exercício físico para a qualidade de vida dos idosos. RBPFEX-Revista Brasileira de Prescrição e Fisiologia do Exercício, 2009; 3(18).

24. MOURA DJM, et al. Cuidado de enfermagem ao cliente com hipertensão: uma revisão bibliográfica. Revista Brasileira de Enfermagem, 2011; 64(4): 759-765.

25. NETTO RORF. Efeitos colaterais dos anti-hipertensivos mediante a prática da atividade física. Revista Magsul de Educação Física na Fronteira, 2017; 2(3): 14-20.

26. NOGUEIRA IC, et al. Efeitos do exercício físico no controle da hipertensão arterial em idosos: uma revisão sistemática. Revista Brasileira de Geriatria e Gerontologia, 2012; 15(3): 587-601.

27. ONYSKO J, et al. Grande aumento no diagnóstico e tratamento da hipertensão no Canadá após um programa de educação profissional em saúde. Hipertensão, EUA, 2006; 48(5): 853-860, conjunto.

28. PÓVOA R. Minieditorial: Características Clínicas da Hipertensão Arterial Resistente versus Refratária em uma População de Hipertensos Afrodescendentes. Arquivos Brasileiros de Cardiologia, 2020; 115(1): 40-41.

29. SANTA-HELENA ET, et al. Fatores associados à não-adesão ao tratamento com anti-hipertensivos em pessoas atendidas em unidades de saúde da família. Caderno de Saúde Pública 2010; 26(12).

30. SANT'ANA DOM. Hipertensão arterial sistêmica: uma proposta de intervenção para o aumento da adesão ao tratamento. 2017.

31. SANTOS ZMSA, LIMA HP. Tecnologia educativa em saúde na prevenção da hipertensão arterial em trabalhadores: análise das mudanças no estilo de vida. Texto \& Contexto-Enfermagem, 2008; 17(1): 90-97.

32. SILVA APD, LARA RT. Perfil epidemiológico dos pacientes hipertensos atendidos em um ambulatório de cardiologia de uma universidade do extremo sul catarinense no período de 2017. 2019.

33. TOLEDO MM, et al. Educação em saúde no enfrentamento da hipertensão arterial: uma nova ótica para um velho problema. Texto \& Contexto-Enfermagem, 2007; 16(2): 233-238. 